\title{
Mechanism of action of interferon-tau in the uterus during early pregnancy
}

\author{
T. R. Hansen ${ }^{1}$, K. J. Austinn ${ }^{1}$, D. J. Perry ${ }^{1}$, J. K. Pru', \\ M. G. Teixeira ${ }^{2}$ and G. A. Johnson ${ }^{1+}$ \\ ${ }^{2}$ Department of Animal Science and ${ }^{2}$ School of Pharmacy, Reproductive Biology Progran, \\ University of Wyoming, Laramie, WY 82071, USA
}

\begin{abstract}
Early pregnancy is maintained in ruminants through the actions of conceptus-derived interferon (IFN)-tau on the endometrium. IFN-tau alters uterine release of $\mathrm{PGF}_{20^{\prime}}$ which results in rescue of the corpus luteum and continued release of progesterone. The mechanism of action of IFN-tau includes inhibition of oestradiol receptors, consequent reduction in oxytocin receptors, activation of a cyclooxygenase inhibitor, and a shift in the PGs to favour $\mathrm{PGE}_{2}$ over $\mathrm{PGF}_{2 \alpha^{\circ}}$. IFN-tau also induces several endometrial proteins that may be critical for survival of the developing embryo. One endometrial protein induced by pregnancy and IFN-tau has been identified as bovine granulocyte chemotactic protein-2 (bGCP-2). This chemotactic cytokine (chemokine) has been used as a marker to delineate IFN-tau from IFN-alpha responses in the endometrium. A second protein, called ubiquitin cross-reactive protein (UCRP), resembles a tandem ubiquitin repeat. UCRP becomes conjugated to cytosolic endometrial proteins in response to IFN-tau and pregnancy. Proteins conjugated to UCRP are either modulated or targeted for processing through the proteasome. The action of IFN-tau is mediated by induction of signal transducer and activator of transcription 1 (STAT-1), STAT-2 and interferon regulatory factor 1 (IRF-1) transcription factors. Induction of these transcription factors, the alpha chemokines and UCRP is the prelude to maternal recognition of pregnancy in ruminants.
\end{abstract}

\section{Introduction}

The mechanism through which interferon-tau (IFN-tau) alters release of the prostaglandins (PGs) during early pregnancy in cows and ewes will be discussed in this review. However, the primary focus will be description of induction of endometrial bovine granulocyte chemotactic protein-2 (bGCP-2) and ubiquitin crossreactive protein (bUCRP) by pregnancy and IFN-tau. Bovine UCRP is induced by IFN-alpha and IFN-tau, whereas bGCP-2 is induced only by IFN-tau. The mechanism of IFN-tau action includes induction of signal transducers and activators of transcription (STAT) and IFN regulatory factor 1(IRF-1) in the bovine endometrium. However, IFN-tau-specific induction of GCP-2 may involve a variant receptor subunit that is associated with the janus kinase (Jak)/STAT/IRF, as well as protein kinase $C$ signal transduction pathways. An overview of function of ubiquitin and human IFN-stimulated gene product 15 (huISG15) is provided as a basis for understanding the function of bUCRP in conjugating to and regulating endometrial proteins during early pregnancy in cows.

\section{Luteolysis}

The oxytocin receptor is a seven transmembrane, G-protein-associated receptor that induces inositol triphosphate turnover, cytosolic calcium and protein kinase C (reviewed in Flint et al., 1995). In the

IPresentaddress: Department of Animal Science, Texas A \& M University, College Station TX 77843. 
uterus, these events result in activation of cyclooxygenase 2 (COX-2) which is the inducible form of PG synthase (Asselin et al., 1997a). The resulting increase in $\mathrm{PGF}_{20}$ causes regression of the corpus luteum. Destruction of the corpus luteum by $\mathrm{PGF}_{2 \alpha}$ results in a decline in circulating progesterone. This decline in progesterone primes the hypothalamus and pituitary to release gonadotrophins that induce ovulation and initiate a new oestrous cycle.

It is currently accepted that the oxytocin receptor becomes uncoupled from associated signal transduction pathways during maternal recognition of pregnancy (reviewed in Bazer et al., 1997). The result is an attenuation of release of $\mathrm{PGF}_{2 n^{\prime}}$, rescue of the corpus luteum, continued release of progesterone, and uterine support of the developing embryo. The hormone or cytokine implicated in uncoupling the oxytocin receptor during pregnancy in ruminants is conceptus-derived IFN-tau (reviewed in Thatcher et al. 1995; Bazer et al., 1997).

\section{Interferon-tau}

Interferon-tau, originally called trophoblastin or trophoblast protein-1, was identified as dominant polypeptides that were released by the conceptus on days 16-24 of pregnancy (reviewed in Roberts et al, 1990). Infusion of these trophoblast proteins into uteri of non-pregnant cows caused an extension of the oestrous cycle that was mediated by the attenuation of release of $\mathrm{PGF}_{2 a}$. Isolation of the cDNA encoding the bovine trophoblast polypeptide and inferred amino acid sequence revealed $45-70 \%$ identity with type I IFNs $(\alpha, \beta, \omega)$ (Imakawa et al., 1990). The trophoblast IFNs were subclassified as IFN-tau in 1990 (Roberts et al.) because of differences in cDNA sequence (Imakawa et al., 1990), number of genes (Hansen et al., 1991), binding to receptors (Hansen et al., 1989), organization of promoters on genes (Hansen et al., 1991), trophoblast-specific expression (Farin et al., 1990), and distribution of genes across species.

\section{Interferon Receptor and Signal Transduction}

Interferon-tau binds to type I IFN receptors that are present in the uterine endometrium (Hansen $e t$ al., 1989; Han et al., 1997). The subunits of IFN-alpha receptors have been named IFNARI and IFNAR2, and co-expression is necessary for functional high affinity IFN binding and signal transduction (Domanski et al., 1995). The extracellular region of IFNAR1 consists of two distinct 200 amino acid domains (D200).

Janus kinase-1 (JAK-1) and tyrosine kinase-2 (Tyk-2) constitutively associate with IFN receptors. Tyrosine kinase-2 associates with the cytoplasmic domains of both IFNAR1 and IFNAR2 (Colamonici et al., 1994), while Jak-1 associates preferentially with IFNAR2 (Novick et al., 1994). It is believed that these tyrosine kinases directly phosphorylate STAT proteins, although other kinases may be involved (Darnell, 1997). STATs undergo tyrosine phosphorylation, assemble into multimeric complexes, of which IFN-stimulated gene factor 3 (ISGF3) is an example, and translocate to the cell nucleus. ISGF3 is composed of a $48 \mathrm{kDa}$ DNA-binding protein termed p48, STAT-1a (p91), STAT-1b (p84), and STAT-2 (p113) (Kessler et al., 1990). After transport to the nucleus, ISGF3 specifically recognizes the IFN-stimulated response element (ISRE) DNA sequence (Levy et al., 1989). Although a consensus ISRE sequence has been defined, there is considerable sequence heterogeneity. Together, differential activation and combinatorial association of these transcription factors regulate IFN-stimulated genes (ISGs).

\section{IFN-tau and the Oxytocin Receptor}

Regulation of the oxytocin receptor is an essential event in the mechanism of action of IFN-tau. In pregnant ewes, it is likely that IFN-tau attenuates release of $\mathrm{PGF}_{2 a}$ by inhibiting formation of oxytocin receptors (Vallet et al., 1990). Progesterone, oestrogen, oxytocin, and their respective 


\begin{tabular}{|c|c|c|c|c|c|c|c|}
\hline & 10 & 20 & 30 & 40 & 50 & 60 & 70 \\
\hline bGCP - 2 & VAAVVRELR & CVCLTTTPG & IHPKTVSDLQ & VIAAGPQCSK & VEVIATLKNG & REVCLDPEAP & LIKKIVQKIL \\
\hline $8 K-9$ & $(100 \%)$ & & TVSDLQ & VIAAGPQ & & & \\
\hline $8 K-13$ & (92\%) & & & & & EVCLXPEAP & LIK \\
\hline
\end{tabular}

MMIP-2 AVVASEL RCQCLKTLPG IDLKNIQSLS VTPPGPHCAQ TEVIATLKGG QKVCLDPEAP LVQKIIQKIL NKGKAN $8 \mathrm{~K}-10 \quad\left(88^{\circ} \mathrm{O}\right)$

$8 \mathrm{~K}-12 \quad(67 \%)$ TPPGPHSgQ TEVIATL

Fig. 1. Amino acid sequence of peptides derived from the purified uterine $8 \mathrm{kDa}$ protein and identity with the alpha chemokines: bovine granulocyte chemotactic protein 2 (bGCP-2) and murine macrophage inflammatory protein 2 (mMIP-2). The $8 \mathrm{kDa}$ protein was purified and digested to yield peptides $(8 \mathrm{~K}-9$, $8 \mathrm{~K}-13,8 \mathrm{~K}-10$ and $8 \mathrm{~K}-12$ ) that were partially sequenced. Peptide amino acids and identities (in parentheses) are shown underneath amino acid sequences for bGCP-2 and mMIP-2. Amino acid sequences have been reported previously for the $8 \mathrm{~K}-9$ and $8 \mathrm{~K}-13$ peptides (Teixeira et al., 1997).

receptors are involved in this process. The theory is that progesterone blocks oestrogen-induced oxytocin receptor synthesis immediately before luteolysis (McCracken et al., 1984). Interferon-tau continues to inhibit oestrogen receptors and oxytocin receptors during early pregnancy, resulting in attenuation of pulsatile PGF $_{2 a}$ release. In sheep, IFN-tau suppresses oestrogen receptor gene expression in the endometrial epithelium (Spencer and Bazer, 1996), which in turn inhibits oxytocin receptor formation and oxytocin-induced signal transduction through inositol triphosphate turnover (Mirando et al., 1990; Ott et al., 1992). These effects on the oxytocin receptor, coupled with activation of a PG synthase inhibitor in cows (Thatcher et al, 1995), result in attenuation of luteolytic $\mathrm{PGF}_{2 \alpha}$ pulses. In addition to inhibiting $\mathrm{PGF}_{2 \mathrm{a}}$ release, IFN-tau also might stimulate release of $\mathrm{PGE}_{2}$ at the expense of $\mathrm{PGF}_{2 \alpha}$ in cows (Asselin et al., 1997b). This concept is intriguing in the context of the luteotrophic effects of $\mathrm{PGE}_{2}$ and in regard to how synthesis of PGs might be regulated by IFN-tau during early pregnancy. IFN-tau might induce degradation or activation of an enzyme downstream from COX-2 (i.e., $\mathrm{PGE}_{2}-9$-ketoreductase) that favours synthesis of $\mathrm{PGE}_{2}$ rather than $\mathrm{PGF}_{2 \mathrm{i}}$ in endometrial epithelial cells. It also might activate transcription factors that inhibit, or inhibit transcription factors that activate ${ }_{x}$ the oestrogen receptor gene.

\section{IFN-tau and Uterine Proteins}

\section{Uterine chemokines}

IFN-tau induces $8 \mathrm{kDa}$ uterine proteins during early pregnancy in cows (Rueda et al., 1993). Amino acid sequencing of four internal peptides derived from the $8 \mathrm{kDa}$ uterine proteins revealed identity with the alpha chemokine family: $92-100 \%$ identity with bovine bGCP-2 (Teixeira et al., 1997), and 67-88\% identity with murine $(\mathrm{m})$ macrophage inflammatory protein-2 (mMIP-2; Fig. 1). Antiserum against bGCP-2 peptide was generated and used in western blot studies to describe release of bGCP-2 by the endometrium during early pregnancy and in response to conceptusderived IFN-tau (Fig. 2). Bovine GCP-2 was not released by endometrium representing the oestrous cycle or in response to the closely related IFN-alpha unless high concentrations were used (Fig. 2b; Staggs et al., 1998). This unique induction of bGCP-2 prompted the hypothesis that it could be used as an index of IFN-tau-specific signal transduction. Another interesting aspect was that phorbol ester mimicked the effects of IFN-tau in inducing the release of bGCP-2 by cultured bovine primary endometrial cells (BEND cells) (Staggs et al., 1998). It was concluded from these experiments that IFN-tau signal transduction was complex in the bovine endometrium and might involve transcription factors other than or ancillary to those described for the Jak/STAT pathway. Type I IFNs compete for binding to a common cell surface receptor. The AR1 subunit of the IFN receptor undergoes ligand-dependent tyrosine phosphorylation. It is possible that AR1 undergoes a specific conformational change in response to IFN-tau to allow phosphorylation of transcription factors that are unique to IFN-tau-specific signal transduction. 

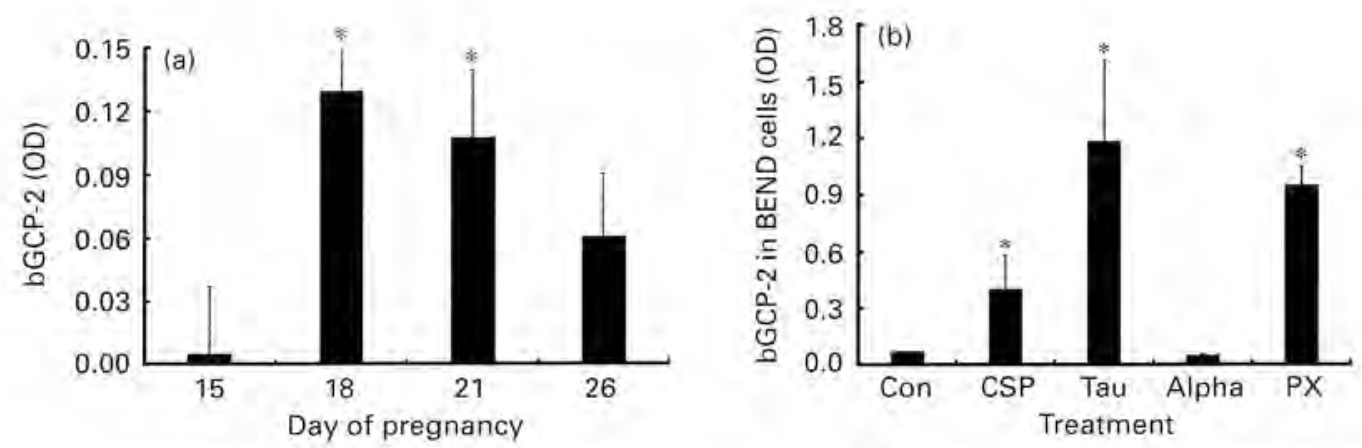

Fig. 2. Release of bovine granulocyte chemotactic protein $2(\mathrm{bGCP}-2)$ in response to pregnancy (a) and conceptus secretory protein (CSP; $200 \mu \mathrm{g} \mathrm{ml}^{-1}$ ), IFN-tau (Tau; $25 \mathrm{nmol} \mathrm{l^{-1 }}$ ), IFN-alpha (Alpha; $25 \mathrm{nmol} \mathrm{I}^{-1}$ ) and phorbol ester (PX;100 $\mathrm{ng} \mathrm{ml}^{-1}$ ), Con: control (b). In (a), explants were collected from pregnant cows on days $15,18,21$, or 26 of pregnancy $(n=3$ cows per day) and cultured for 24 h as described by Austin et al. (1996a). In (b), bovine endometrial (BEND) cells ( $n=3$ replicates) were cultured for $24 \mathrm{~h}$ with respective treatments as described by Staggs et al. (1998). Bovine GCP- 2 was detected using one-dimensional PAGE and western blot analysis (anti-boGCP-2 peptide antiserum; Teixeira et al., 1997). Western blots were scanned using densitometry. ${ }^{*}$ Optical density (OD) means were significantly different $(P<0.05)$ when compared with day 15 of pregnancy (a) or controls (b). Data in (b) were redrawn from Staggs et al. (1998) with permission from Biology of Reproduction,

Several uterine proteins are induced by IFN-tau, but only a few of these proteins have been identified. For example, the interferon-induced protein Mx (Charleston and Stewart, 1993), 2', 5' oligoadenylate synthase (Short et al., 1991), and $\beta_{2}$-microglobin (Vallet et al, 1991) are induced by IFN-tau. However, the specific function of these proteins during early pregnancy remains elusive. The chemokines, although specific markers for IFN-tau action, also belong to this growing category of identified proteins with no known specific function during early pregnancy.

Pregnancy is dependent upon a receptive uterus that allows attachment and invasion of the conceptus while preventing immunological rejection. Chemokines are potent chemo-attractants for cells of the immune system and have been implicated in cell adhesion, inflammatory, and angiogenic processes (reviewed in Oppenheim et al, 1991). The chemokines may attract the conceptus or cells of the immune system to implantation sites. They also may direct an immunostimulatory phenotype that, coupled with scavenger effects (macrophage, neutrophils), controls inflammation and adhesion associated with limited invasion of the maternal caruncles by the conceptus. Available recombinant bovine uterine chemokines would facilitate experiments designed to examine these hypotheses.

\section{Ubiquitin crossreactive protein}

A $17 \mathrm{kDa}$ uterine protein was identified through examining proteins released into medium of cultured endometrial explants from day 18 pregnant cows (Naivar et al. 1995). This protein was released by endometrial explants in response to both recombinant ( $\mathrm{r}$ )bIFN-tau and rbIFN-alpha. Because the $17 \mathrm{kDa}$ protein was similar in size to huISG15 (also called huUCRP), and immunoreacted with antiserum against ubiquitin, it was called bUCRP (Austin et al., 1996a). Antiserum against ubiquitin was used to demonstrate that bUCRP was released by the endometrium at times coincident with IFN-tau release from the conceptus. Bovine UCRP was first detected in the medium of cultured endometrial explants representing day 15 of pregnancy. Release of bUCRP increased on day 18 and remained high through day 26 of pregnancy. Recombinant bIFNtau induced release of bUCRP by endometrial explants representing day 12 of the oestrous cycle in a dose-dependent manner. Likewise, bUCRP was found in significant amounts in uterine flushings from day 18 pregnant cows. 
IFN induces release of ISG15 by mouse Ehrlich ascites tumour cells (Farrell et al., 1979). In addition, ISG15 is released by several cell lines in response to IFN and during the acquisition of an antiviral state. Human ISG15 is released in response to type I IFNs and it induces release of IFN-gamma by T-and B-lymphocytes (Recht et al, 1991). Through inducing release of IFN-gamma from T-cells, ISG15 may augment natural killer cell proliferation and activate monocytes and macrophages.

Endometrial UCRP may be the bovine counterpart to huISG15. Bovine UCRP is released by the endometrium in response to IFN-tau during early pregnancy. Whether bUCRP induces IFN-gamma remains to be determined. The activation of natural killer cells by bUCRP in the endometrium is intriguing, as these effects might be considered detrimental to the developing conceptus (Robertson et al, 1994). However, an extensive description of cell-mediated and humoral cytokines, and consequences of action in the bovine endometrium during early pregnancy is lacking. Bovine UCRP has limited amino acid sequence identity with ubiquitin and huISG15, but retains the C-terminal amino acids, Arg-Gly-Gly (RGG) that have been implicated in the first step in covalent conjugation to cytosolic proteins (Austin et al., 1996b). Because bUCRP retains functional amino acids of ubiquitin and is released from endometrial explants in response to IFN-tau during early pregnancy, it probably has cytosolic and extracellular roles that are consistent with the maintenance of pregnancy.

Ubiquitination. Proteolysis is an important intracellular regulatory mechanism for controlling many biological processes. In eukaryotes, ubiquitin-dependent proteolysis is essential for protein turnover. Ubiquitin is a highly conserved 76 amino acid polypeptide that is involved in a wide array of biological systems including cell cycle regulation, DNA repair, receptor modification, signal transduction, antigen presentation, the stress response, and both proteasomal and non-proteasomal protein degradation (Finley and Chau, 1991). Conjugation of ubiquitin to proteins can result in either polyubiquitination or mono-ubiquitination. Polyubiquitination is believed to target proteins for rapid degradation (Finley and Chau, 1991): for example, it may be involved in the process of luteal regression (Murdoch et al., 1996). Carboxyl terminal amino acids on ubiquitin, via the RGG sequence, link to primary amines on targeted proteins (Hershko and Ciechanover, 1992). Packed multi-ubiquitin chains are then added through isopeptide bonds between carboxyl terminals and Lys- 48 residues on successive ubiquitin monomers. This quaternary structure is unique and aids in directing the complex to the proteasome where the targeted protein is degraded and ubiquitin monomers are released and recycled (Finley and Chau, 1991).

Mono-ubiquitinated proteins, such as actin, histones, microtubules and the growth hormone receptor are longer-lived and probably undergo ubiquitin protein modification as well as modest rates of targeted degradation (reviewed in Finley and Chau, 1991). A case in point is the transcription factor nuclear factor kappa B (NF-kB), for which the proteasome proteolytic pathway is required for proper activation (Palombella et al., 1994). This transcription factor regulates a variety of genes involved in immune system function such as those encoding immunoglobulin kappa light chains, interleukin 2 (IL-2) receptor alpha chain, class I major histocompatibility complex (MHC), and a number of cytokines that include IL-2, IL-6, granulocyte-macrophage colony-stimulating factor (GM-CSF), and IFN-beta. NF-kB also has been implicated in the expression of cell adhesion genes that encode selectin, intercellular adhesion molecule 1 and viral cellular adhesion molecule 1 (Palombella et al, , 1994).

Unique primary structure of bUCRP. Human ISG15 has 29-31\% amino acid sequence identity with two tandem ubiquitin monomers. Inferred amino acid sequence of the bUCRP CDNA and gene have $68 \%$ identity with huISG15 and 31\% identity with a tandem ubiquitin repeat (Austin et al., 1996b). Only one major bUCRP gene exists (Perry et al., 1997). Thus, it appears likely that bUCRP is the counterpart to huISG15. However, there are significant differences in primary structure between huISG15 and bUCRP. The huISG15 C-terminal amino acids are removed via post-translational processing to yield a $15 \mathrm{kDa}$ protein that ends in RGG (Feltham et al., 1989). The bUCRP CDNA 
encodes a $17 \mathrm{kDa}$ protein in which translation stops immediately after RGG amino acids (Austin et al., 1996b). The coding region of the bUCRP gene is identical to that reported for the CDNA. Functional residues of ubiquitin that are retained in bUCRP include the C-terminal RGG residues and Arg-72 of ubiquitin that have been retained as Arg-150 in bUCRP. This residue is involved with binding to ubiquitin conjugating enzyme (E1), and is required for ordered addition of substrate in a complex ligation pathway (Burch and Haas, 1994). Other ubiquitin residues implicated in binding to targeted proteins (i.e., Arg54), degradation through the $26 \mathrm{~S}$ proteasome (i.e. His68) (Ecker et al., 1987), and polymerization of monomers (Lys48) (Finley and Chau, 1991) are absent in bUCRP.

Unique cytosolic role of bUCRP. It was hypothesized that bUCRP has a cytosolic role in the modification of uterine proteins during early pregnancy. In a manner similar to ubiquitin, bUCRP may become conjugated to cytosolic endometrial proteins. Because bUCRP lacked residues known to form ubiquitin polymers and to target proteins for degradation, it was suspected that the array of proteins conjugated to bUCRP, and the destiny of these proteins might be different from those conjugated to ubiquitin.

Antiserum was developed against a bUCRP peptide that immunoreacted with bUCRP, but did not immunoreact with ubiquitin on western blots. This antibody also detected bUCRP when conjugated to an array of endometrial proteins (Johnson et al., 1998). These complexes with bUCRP were called conjugates and represented proteins coupled to a single bUCRP molecule (monomer), or to polymers of bUCRP. Co-incubation of antibody against bUCRP with antigenic bUCRP peptide blocked detection of bUCRP and its conjugates. Pre-absorption of bUCRP antibody with ubiquitin had no effect on detection of bUCRP or bUCRP conjugates. Thus, detection of bUCRP and its conjugates by the anti-bUCRP antibody was immunospecific.

Ubiquitin and its conjugates are abundant in the bovine endometrium and are not regulated by IFN-tau (Johnson et al., 1998). Because bUCRP and its conjugates were induced by pregnancy and IFN-tau (Fig. 3a), it was concluded that proteins conjugated to bUCRP were distinct from those conjugated to ubiquitin. The induction of bUCRP and its conjugates in endometrial explants required exposure to rbIFN-tau for $12 \mathrm{~h}$. This appearance of bUCRP and its conjugates was consistent with the time required for transcription, translation, and conjugation of bUCRP to cytósolic proteins.

Phosphorylated STATs become ubiquitinated and degraded within minutes after treatment with IFN-gamma (Kim and Maniatis, 1996). This process is so fast that use of proteasome inhibitors was required to observe an accumulation of ubiquitinated STAT-1. Interestingly, proteins conjugated to bUCRP continued to accumulate through $48 \mathrm{~h}$ after treatment with rbIFN-tau (Johnson et al., 1998). This accumulation of proteins that were conjugated to bUCRP might reflect increased protein modulation or stabilization as opposed to degradation. It was inferred from these experiments that regulation or degradation of endometrial proteins by bUCRP was a component of establishing early pregnancy in ruminants. For example, bUCRP might ligate to and modify, or initiate proteasomal degradation of cytosolic uterine proteins that are involved with the release of $\mathrm{PGF}_{2 \mathrm{n}}$.

IFN-tau may induce conjugation of bUCRP to the oxytocin receptor so that it becomes unable to bind to oxytocin during early pregnancy in cows (Fig. 4). Alternatively, bUCRP may conjugate to the oestrogen receptor, or to transcription factors that induce transcription of the oestrogen receptor gene and target these proteins to degradation. Likewise, enzymes involved with the synthesis of PGs may be modified by bUCRP such that $\mathrm{PGF}_{2 \mathrm{n}}$ is attenuated and $\mathrm{PGE}_{2}$ is stimulated. In addition, IFNtau may dictate the selective proteolysis or modulation of uterine cytokines by bUCRP to accommodate the changing immune demands of pregnancy. The identification of proteins that are conjugated to bUCRP during early pregnancy and in response to IFN-tau would address these hypothesized roles of bUCRP.

Induction of bUCRP $m$ RNA in the endometrium by IFN-tau. Another major difference between huISG15 and bUCRP is the apparent widespread expression of the huISG15 gene in several cell lines and tissues (Lowe et al., 1995). Northern blot analysis revealed that expression of the bUCRP gene was 
(a)

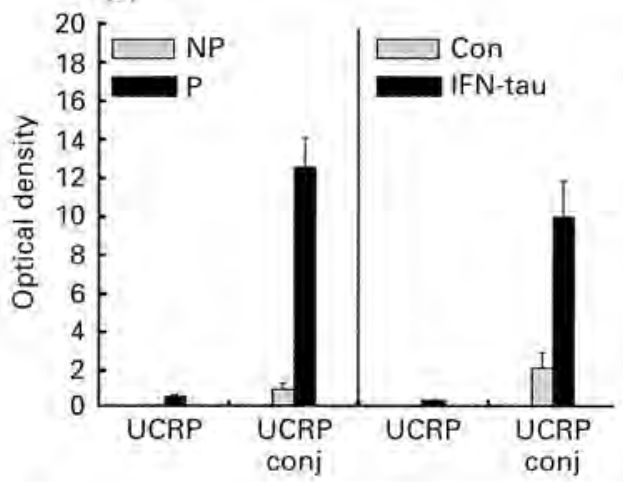

(b)

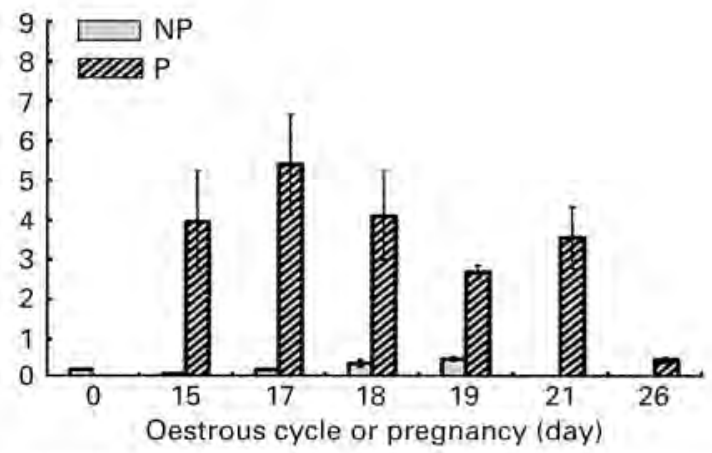

Fig. 3. Induction of bovine ubiquitin crossreactive protein (bUCRP) in endometrial explants. (a) Induction of bUCRP and its conjugates by pregnancy and IFN-tau. Explants from day 18 pregnant $(\mathrm{P} ; n=4)$ or nonpregnant (NP, $n=4$ ) cows were cultured for $24 \mathrm{~h}$. Cytosolic proteins were separated using one-dimensional PAGE and analysed for the presence of bUCRP and its conjugates using western blotting as described by Johnson et al. (1998). Endometrial explants from day 14 non-pregnant cows $(n=3)$ were cultured in the absence (Con) or presence $\left(25 \mathrm{nmol} \mathrm{l}^{-1}\right)$ of rbIFN-tau for $24 \mathrm{~h}$. Bovine UCRP and its conjugates were detected as described in Johnson et al. (1998). Data in (a) are adapted from Johnson et al. (1998) with permission from Biology of Reproduction. (b) Endometrium was collected from non-pregnant and pregnant cows on the days specified ( $n=4$ cows on each day). RNA was isolated, northern blotted, hybridized with radiolabelled bUCRP CDNA and quantitated as described in Hansen et al. (1997); (b) is reprinted from Hansen et al. (1997) with permission from The Endocrine Society. ${ }^{*}$ Optical density means were significantly different $(P<0.05)$ from NP or Con.

restricted to endometria from pregnant cows or from endometria from non-pregnant cows that had been treated in vitro with rbIFN-tau (Hansen et al., 1997).

Bovine UCRP mRNA was not detected using northern blot analysis in endometrium from nonpregnant cows (days 15,17, 18 or 19) or in spleen, kidney, liver, corpus luteum or muscle (Hansen et al., 1997). The bUCRP cDNA did not hybridize to ubiquitin mRNAs. Both major ubiquitin transcripts $\mathrm{UbB}(1.2 \mathrm{~kb})$ and $\mathrm{UbC}(2.5 \mathrm{~kb})$ were present in all tissues examined and were not regulated by IFNtau. Bovine UCRP mRNA was detected in endometria from pregnant cows by day 15 , reached highest values by day 17 , remained high on days 18,19 and 21 , and then declined to amounts that were not detectable on day 26 (Fig. 3b). This pattern of transcription closely paralleled detection of UCRP protein in cultured endometrial explants from cows on the same days of pregnancy (Austin et al., 1996a). The bUCRP mRNA transcript ( 700 bp) can be induced in bovine endometrial cells (i.e. BEND cells) derived from non-pregnant cows when cultured with $25 \mathrm{nmol} \mathrm{rbIFN-tau} \mathrm{I}^{-4}$ (Hansen et al., 1997). Although the role of huISG15 may be general to many cell types, transcription of the bUCRP gene appears to be a specific uterine response to conceptus-derived IFN-tau during early pregnancy in cows.

The bovine UCRP gene. The huISG15 promoter contains a functional tandem ISRE at position -39 relative to the mRNA cap site (Reich et al., 1987), but little is known about sequences that are upstream of this tandem ISRE. The TATA box (TATTAAA) in the bUCRP gene is located at position -31 from the mRNA cap site (Perry et al., 1997). Both genes contain a single intron after the initiation codon, followed by coding, termination, $3^{r}$ non-coding, and polyadenylation sequences. The bUCRP gene promoter is similar to the huISG15 gene promoter in the placement of a conserved tandem ISRE at position -90. However, three additional putative ISREs are present in the bUCRP gene at positions $-123,-332$ and -525 . 

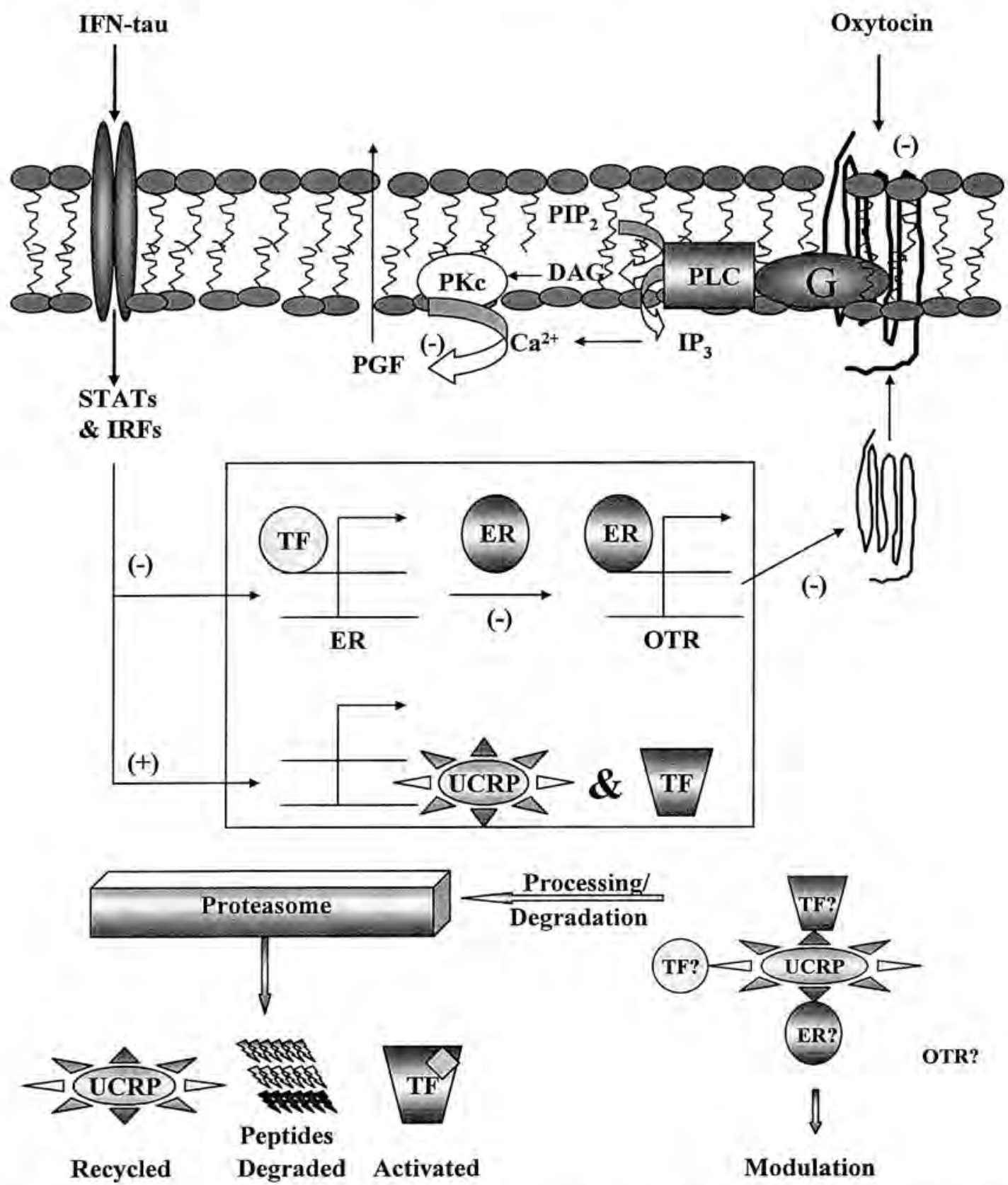

Fig. 4. Hypothetical mechanism of IFN-tau action in bovine uterine endometrium. IFN-tau binds to an endometrial receptor and induces phosphorylation of signal transducers and activators of transcription (STATs). STATs induce transcription and subsequent phosphorylation of interferon regulatory proteins (IRFs). The STATs and IRFs may have direct actions through inhibiting the oestradiol receptor (ER), and potentially, the oxytocin receptor (OTR) genes. Disruption of oxytocin receptor gene transcription results in reduced binding to oxytocin. Consequently, G proteins (G), phospholipase C (PLC), second messengers associated with activation of protein kinase $\mathrm{C}(\mathrm{PKc})$, and increased calcium become uncoupled resulting in diminished release of $\mathrm{PGF}_{30^{\circ}}$. In addition to inhibiting transcription of the oestradiol receptor gene, IFN-tau, through inducing the STATs and IRF-1 also induces transcription of bovine ubiquitin crossreactive protein (bUCRP) and potentially transcription factor (TF) 
Interferon receptors exhibit preference for ligand and sort cytosolic responses through tyrosine kinase phosphorylation of at least six STAT transcription factors. Nuclear proteins induced by rbIFN-tau in BEND cells interact with bUCRP ISRE DNA in mobility shift assay (Perry et al., 1998). At least four slower migrating bands were induced by rbIFN-tau. IFN-tau induced appearance of STAT-1, STAT-2 and IRF-1 in nuclear proteins extracted from BEND cells (Perry et al., 1998; Binelli ef al., 1998). The temporal relationship between appearance of the STATs $(0.5 \mathrm{~h})$ and IRF-1 $(2 \mathrm{~h})$ in BEND cells was consistent with induction of these nuclear proteins in other cells by other type I IFNs. The STATs are activated within minutes of IFN treatment. Induction of phosphorylation of cytoplasmic STATs initiates translocation to the nucleus, formation of transcriptional complexes and induction of secondary transcription factors called IRFs. The bUCRP gene may be induced by STATs and IRF-1. The intriguing and more complex issue raised by these experiments is how the STATs and IRF-1 regulate other genes in the endometrium during early pregnancy. Genes involved with the conjugation of bUCRP to targeted cytosolic proteins may be induced by these transcription factors. Likewise, genes encoding enzymes that shunt arachidonic acid to PGF may be inhibited by these transcription factors.

\section{Conclusions}

The study of uterine responses to the conceptus during early pregnancy resulted in identification of several uterine proteins that may prepare the uterus for the implanting embryo. One critical event that must be circumvented or altered is uterine release of $\mathrm{PGF}_{2 a}$ (Fig. 4). Inhibition of transcription of the oestrogen receptor gene is one critical action of IFN-tau in the modulation of $\mathrm{PGF}_{2 a}$ release (Spencer and Bazer, 1996). Exactly how IFN-tau regulates the oestrogen receptor gene remains to be determined. It may involve direct interaction of the STATs or IRFs (Bazer et al., 1997; Spencer et al., 1998) with negative acting response elements. Alternatively, it may involve removal of positive trans-acting transcription factors through conjugation to bUCRP and degradation through the proteasome. Likewise, enzymes involved in the synthesis of $\mathrm{PGF}_{2 a}$ could be degraded through conjugation to bUCRP, whereas enzymes involved in the synthesis of $\mathrm{PGE}_{2}$ could be activated or stabilized.

Alpha chemokines have only recently been identified in the uterine endometrium (Teixeira et al., 1997). The function of these chemotactic cytokines in the uterus is unknown, but may involve preparing the endometrium for implantation or changing the immune environment to an environment that nurtures survival of the embryo. Several years ago it was suggested that the IFNtau receptor was complex based on curvilinear Scatchard plot and cross-linking experiments. (Hansen et al. 1989). Recent experiments, using cultured endometrial explants and BEND cells, revealed that the uterine endometrium can distinguish between type I IFNs (Staggs et al., 1998). Phorbol ester and IFN-tau, but not the closely related IFN-alpha, induced release of bGCP-2. However, the IFN receptor cloned from ovine endometrium appears to be identical in nucleotide sequence to the IFN-alpha receptor (Han et al, 1997). Because this IFN receptor was cloned using IFN-alpha receptor sequences, it may not be surprising that the receptor identified in ovine and bovine endometrium was identical. It remains unresolved whether there is a unique IFN-tau receptor subunit in the endometrium. Likewise it remains unknown if the IFNAR might change conformation after binding IFN-tau to expose a cytosolic domain that activates IFN-tau-specific STATs or IRFs. IFN-tau signal transduction appears to involve the STATs, and IRF-1. Future experiments using the bUCRP gene promoter and potentially the bGCP-2 gene promoter may delineate exactly which transcription factors are induced in response to IFN-tau. Certainly

genes. Bovine UCRP becomes conjugated to cytosolic proteins and either modulates these proteins or targets them to the proteasome where they are either degraded into peptides or activated. An example of activation is provided by the transcription factor, NF-kappa B that must be processed through the proteasome prior to regulating transcription of genes (Palombella et al, 1994). DAG: diacyl glycerol; PIP 2 : phosphoinositol-4,5 diphosphate. 
development of the BEND cell line will facilitate these experiments as well as those planned to identify endometrial proteins that become conjugated to bUCRP in response to pregnancy and IFN-tau.

*This research was supported by grants from the National Institutes of Health (\# HD 32475), United States Department of Agriculture (\# 97-35203-4808), and University of Wyoming Agricultural Competitive Grants Program.

\section{References}

Asselin E, Drolet P and Fortier MA (1997a) Cellular mechanisms involved during oxytocin-induced prostaglandin $\mathrm{F}_{7 \mathrm{o}}$ production in endometrial epithelial cells in vitro: role of cyclooxygenase-2 Endocrinology $1384798-4805$

Asselin E, Bazer FW and Fortier MA (1997b) Recombinant ovine and bovine interferons tau regulate prostaglandin production and oxytocin response in cultured bovine endometrial cells Biology of Reproduction $56402-408$

Austin KJ, Ward SK, Teixeira MG, Dean VC, Moore DW and Hansen TR (1996a) Ubiquitin cross-reactive protein is released by the bovine uterus in response to interferon during early pregnancy Biology of Reproduction 54 600-606

Austin KJ, Pru JK and Hansen TR (1996b) Complementary deoxyribonucleic acid sequence encoding bovine ubiquitin cross-reactive protein: a comparison with ubiquitin and a 15-kDa ubiquitin homolog Endocrine 5 191-197

Bazer FW, Spencer TE and Ott TL (1997) Interferon tau: a novel pregnancy recognition signal American loumal of Reproductive Ininunology $37412-420$

Binelli M, Diaz T, Hansen TR and Thatcher WW (1998) Bovine interferon-tau induces phosphorylation and nuclear translocation of STAT-1, -2 , and -3 in endometrial epithelial cells Biology of Reproduction (Supplement 1) 214 (Abstract)

Burch TJ and Haas AL (1994) Site-directed mutagenesis of ubiquitin. Differential roles for arginine in the interaction with ubiquitin-activating enzyme Biochemistry 33 7300-7308

Charleston B and Stewart HJ (1993) An interferon-induced Mx protein: CDNA sequence and high-level expression in the endometrium of pregnant sheep Gene 137 327-331

Colamonici O, Yan H, Domanski P, Handa R, Smalley D, Mullersman J, Witte M, Krishnan K and Krolewski J (1994) Direct binding to and tyrosine phosphorylation of the alpha subunit of the type 1 interferon receptor by 135 tyk 2 tyrosine kinase Molecular and Cellular Biology 14 8133-8142

Darnell JE, Jr (1997) STATs and gene regulation Science 277 $1630-1635$

Domanski P, Witte M, Kellum M, Rubinstein M, Hackett R, Pitha P and Colamonici OR (1995) Cloning and expression of a long form of the interferon alpha beta receptor that is required for signaling Journal of Biological Chemistry 270 21606-21611

Ecker DJ, Butt TR, Marsh J, Sternberg EJ, Margolis N, Monia BP, Jonnalagadda S, Khan Ml, Weber PL, Mueller L and Crooke ST (1987) Gene synthesis, expression, structures, and functional activities of site-specific mutants of ubiquitin Journal of Biological Chemistry 262 14213-14221

Farin CE, Imakawa K, Hansen TR, McDonnell JJ, Murphy CN, Farin PW and Roberts RM (1990) Expression of trophoblastic interferon genes in sheep and cattle Biology of Reproduction $43210-218$

Farrell PJ, Broeze RJ and Lengyel P (1979) Accumulation of an mRNA and protein in interferon-treated Ehrlich ascites tumour cells Nature 279 523-525
Feltham N, Hillman M, Jr, Cordova B, Fahey D, Larsen B, Blomstrom D and Knight E, Jr (1989) A 15-kD interferoninduced protein and its $17-\mathrm{kDa}$ precursor: expression in Escherichia coli, purification, and characterization Journal of Interferon Research 9 493-507

Finley D and Chau V (1991) Ubiquitination Annual Review of Cell Biology 7 25-69

Flint APF, Riley PR, Stewart HJ and Abayasekara DRE (1995) The sheep endometrial oxytocin receptor Advances in Experimental Medicine and Biology 395 281-294

Han CS, Mathialagan N, Klemann SW and Roberts RM (1997) Molecular cloning of ovine and bovine type 1 interferon receptor subunits from uteri, and endometrial expression of messenger ribonucleic acid for ovine receptors during the estrous cycle and pregnancy Endocrinology 138 $4757-4767$

Hansen TR, Kazemi M, Keisler DH, Malathy P-V, Imakawa K and Roberts RM (1989) Complex binding of the embryonic interferon, ovine trophoblast protein-1 to endometrial receptors Journal of Interferon Research 9 215-225

Hansen TR, Leaman DW, Cross JC, Mathialagan N, Bixby JA and Roberts RM (1991) The genes for the trophoblast interferons and the related interferon all possess distinct 5 '-promoter and 3 '-flanking sequences fournal of Biological Chemistry 266 3060-3066

Hansen TR, Austin KJ and Johnson GA (1997) Transient ubiquitin cross-reactive protein gene expression in bovine endometrium Endocrinology 138 5079-5082

Hershko A and Ciechanover A (1992) The ubiquitin system for protein degradation Anmial Revieiv of Biochemistry 61 $761-807$

Imakawa K, Hansen TR, Malathy P-V, Anthony RV, Polites HG, Marotti KR and Roberts RM (1990) Molecular cloning and characterization of complementary deoxyribonucleic acids corresponding to bovine trophoblast protein-1: a comparison with ovine trophoblast protein-1 and bovine interferon- $\alpha_{11}$ Molecular Endocrinology 3 127-139

Johnson GA, Austin KJ, Van Kirk EA and Hansen TR (1998) Pregnancy and interferon-tau induce conjugation of bovine ubiquitin cross-reactive protein to cytosolic uterine proteins Biology of Reproduction 58 898-904

Kessler DS, Veals SB, Fu XY and Levy DE (1990) IFN-alpha regulates nuclear translocation and DNA-binding affinity of ISGF3, a multimeric transcriptional activator Genes and Development 4 1753-1765

Kim TK and Maniatis T (1996) Regulation of interferon- $\gamma$ activated STAT1 by the ubiquitin-proteasome pathway Science 273 1717-1719

Levy DE, Kessler DS, Pine R and Darnell JE (1989) Cytoplasmic activation of ISGF3, the positive regulator of interferonalpha-stimulated transcription, reconstituted in vitro. Genes and Development 3 1362-1371 
Lowe J, McDermott H, Loeb K, Landon M, Haas AL and Mayer RJ (1995) Immunohistochemical localization of ubiquitin cross-reactive protein in human tissues Joumal of Pathology 177 163-169

McCracken JA, Schramm W and Okulicz WC (1984) Hormone receptor control of pulsatile secretion of PGF2a from the ovine uterus during luteolysis and its abrogation in early pregnancy Animal Reproduction Science 7 31-55

Mirando MA, Ott TL, Vallet JL, Davis M and Bazer FW (1990) Oxytocin-stimulated inositol phosphate turnover in endometrium of ewes is influenced by stage of the estrous cycle, pregnancy, and intrauterine infusion of ovine conceptus secretory proteins Biology of Reproduction 42 98-105

Murdoch WJ, Austin KJ and Hansen TR (1996) Polyubiquitin upregulation in corpora lutea of prostaglandin treated ewes Endocrinology $1374526-4529$

Naivar KA, Ward SK, Austin KJ, Moore DW and Hansen TR (1995) Secretion of bovine uterine proteins in response to type-1 interferons Biology of Reproduction 52 848-854

Novick D, Cohen B and Rubenstein M (1994) The human interferon alpha/beta receptor: characterization and molecular cloning Cell $77391-400$

Oppenheim JJ, Zachariae COC, Mukaida $\mathrm{N}$ and Matsushima $\mathrm{K}$ (1991) Properties of the novel proinflammatory supergene "intercrine" cytokine family Annual Review of Immunology $9617-648$

Ott TL, Mirando MA, Davis MA and Bazer FW (1992) Effects. of oyine conceptus secretory proteins and progesterone on oxytocin-stimulated endometrial production of prostaglandin and turnover of inositol phosphate in oyariectomized ewes Journal of Reproduction and Fertility 95 19-29

Palombella VJ, Rando OJ، Goldberg AL and Maniatis T (1994) The ubiquitin-proteasome pathway is required for processing the NF-kappa B1 precursor protein and the activation of NF-kappa B Cell 78 773-785

Perry DJ, Austin KJ and Hansen TR (1997) The uterine bovine ubiquitin cross-reactive protein gene: a comparison with human interferon stimulated gene 15 Biology of Reproduction 56 (Supplement 1) 128 (Abstract 181)

Perry DJ, Austin KJ and Hansen TR (1998) Transcription factors that are involved with the mechanism of interferon-tau action in bovine endometrial cells The Endocrine Society Program Abstracts of the $80^{m}$ Annual Mecting p222

Recht M, Borden EC and Knight E, Jr (1991) A human 15-kDa IFN-induced protein induces the secretion of IFN- Journal of Imminology $1472617-2623$
Reich N, Evans B, Levy D, Fahley D, Knight E, Jr and Darnell JE, Jr (1987) Interferon-induced transcription of a gene encoding a $15-\mathrm{kDa}$ protein depends on an upstream enhancer element Proceedings of the National Academy of Sciences USA $846394-6398$

Roberts RM, Cross JC and Leaman DW (1990) Interferons as hormones of pregnancy Endocrine Revicus $13432-452$

Robertson SA, Seamark RE, Guilbert LJ and Wegmann TG (1994) The role of cytokines in gestation Crifical Reviews in Immunology 14 239-292

Rueda BR, Naivar KA, George EM, Austin KJ, Francis $\mathrm{H}$ and Hansen TR (1993) Recombinant interferon- $\tau$ regulates secretion of two bovine endometrial proteins Jourmal of Interferon Research 13 303-309

Short EC, Geisert RD, Helmer SD, Zavy MT and Fulton RW (1991) Expression of antiviral activity and induction of 2 ', 5'oligoadenylate synthetase by conceptus secretory proteins enriched in bovine trophoblast protein-1 Biology of Reproduction 44 261-268

Spencer TE and Bazer FW (1996) Ovine interferon tau suppresses transcription of the estrogen receptor and oxytocin receptor genes in ovine endometrium Endocrinology 137 1144-1147

Spencer TE, Ott TL and Bazer FW (1998) Expression of interferon regulatory factors one and two in the ovine endometrium: effects of pregnancy and ovine interferon tau Biology of Reproduction 58 1154-1162

Staggs KL, Austin KJ, Johnson GA, Teixeira MG, Talbott CT, Dooley VA and Hansen TR (1998) Complex induction of bovine uterine proteins by interferon-tau Biology of Reproduction 59 293-297

Teixeira MG, Austin KJ, Perry DJ, Dooley VD, Johnson GA, Francis BR and Hansen TR (1997) Bovine granulocyte chemotactic protein-2 is secreted by the endometrium in response to interferon-tau (IFN- $\tau$ ) Endocrine 6 31-37

Thatcher WW, Meyer MD and Danet-Desnoyers G (1995) Maternal recognition of pregnancy Journal of Reproduction and Ferfility Supplement 49 15-28

Vallet JL, Lamming GE and Batten M (1990) Control of endometrial oxytocin receptor and uterine response to oxytocin by progesterone and oestradiol in the ewe Journal of Reproduction and Fertility $90625-634$

Vallet JL, Barker PJ, Lamming GE, Skinner N and Huskisson NS (1991) A low molecular weight endometrial secretory protein which is increased by ovine trophoblast protein-1 is $\beta 2$-microglobulin-like protein Joumal of Endocrinology 130 R1-R4 